$7^{\text {th }}$ Jagna International Workshop (2014)

International Journal of Modern Physics: Conference Series

Vol. 36 (2015) 1560010 (5 pages)

(C) The Authors

DOI: 10.1142/S2010194515600101

\title{
Robust method of trapping self-propelling particles
}

\author{
Roger Joseph L. Lacubtan \\ College of Arts and Sciences, Central Mindanao University, \\ Musuan, 8710, Philippines \\ Mark Nolan P. Confesor \\ Department of Physics, MSU-Iligan Institute of Technology, \\ Iligan City, 9200, Philippines \\ marknolan2006@gmail.com
}

Published 2 January 2015

\begin{abstract}
The ability to collect self-propelling particles (SPP) is an essential requirement for possible use of SPP in technological applications. In this paper we proposed a novel way of trapping SPP's, through guided trapping of SPP's in V-shaped trap. We performed brownian dynamic simulation via a modified Escape and Predation model developed by L. Angelani (Phys. Rev. Lett., 2012) to assess the validity of the proposed trapping method.
\end{abstract}

Keywords: Collecting self-propelling particles; escape and predation.

\section{Introduction}

Self-propelling particles (SPP) are ubiquitous in nature. SPPs are observed at different length scales; ranging from $\mu m$ sized motile bacteria to $m$ sized fishes such as whales. Due to huge promising application in medicine, nano-technology, and intelligent controls, there is a myriad of artificial SPP's that have been conceived and experimentally made [1].

In most conceived cases of using $\mu m$-sized SPPs in biotechnology, techniques enabling the collection of SPPs are essential. Existing and suggested approaches are the use of funnel shaped gates that leads to the sorting of rod-shaped SPPs to passive ones [2]; use of wedge like obstacles to collect for also rod-like shaped SPPs [3]; and an array of L-shaped obstacles for SPPs moving in circular trajectories [4]. A general mechanism to trap SPPs independent of the shape or the propelling mechanism therefore, has yet to be found.

This is an Open Access article published by World Scientific Publishing Company. It is distributed under the terms of the Creative Commons Attribution 3.0 (CC-BY) License. Further distribution of this work is permitted, provided the original work is properly cited. 
In this study we propose a novel way of trapping SPPs independent of their shape or the nature of the propulsion with shepherding as our inspiration. In our trapping method we make use of another SPP (chaser) to guide our SPP of interest (target) towards a V-shaped trap for collection same as shepherds do to the sheep.

\section{Brownian dynamics simulation}

We performed brownian dynamic simulation of an escape and predation model proposed by L. Angelani [5] but with a modification that the targets are not annihilated and that there is a presence of a V-shaped trap. Furthermore, chasers are not allowed to get inside the space enclosed by the V-shaped trap and such space is accessible only to targets.

In the simulation employed, both the chaser and the target has a constant speed set at $v_{0}$. However, the direction of motion of either the chaser or target can change due to localized interaction. Specifically the velocity of a particle $i$ (target or chaser) is given by [5],

$$
v_{i}^{(i n t)}=\alpha v_{i}^{(a l)}+\beta f_{i}^{(r e p)}+\gamma g_{i}^{(r e p)}+\delta f_{i}^{(a l)} .
$$

The first term corresponds to the alignment of the particles based on the Vicsek model; particle $i$ next position is on the same direction as the average velocity of the neighboring particles (of the same type) within some radius of the particle $i$ [6]. The second and third term in Equation 1, corresponds to the exclusive volume interaction that causes no particle (chaser or target) to be on the same location. The last term corresponds to the chase or escape force such that a chaser will move towards the direction of a target as the target moves away from the chaser. After the calculation of the velocity of each particle, the next position of the particle is updated by $v_{i}^{(i n s t)} \Delta t$, where $\Delta t$ is the time step. Parameter values used in the simulations are similar to those used in Ref. [5].

\section{Escape and predation without annihilation}

To better understand the guided trapping mechanism, first we need to assess the chase statistics for the case when there is no V-shaped trap. In the simulation, we incorporate a repulsive boundary condition and varied the concentration ratio of target and chaser from $0.1 \leq \phi \leq 6$ ( $\phi=$ total number of target/total number of chaser). Screenshot images of the simulation are shown in Figure 1.a-c for three concentration regimes of $\phi$. Initially the particles are distributed randomly in the simulation box. However, as the simulation progress we observe clustering of same particle species. We note that due to the Vicsek alignment, both the target and chaser exhibit collective motion by themselves. Furthermore, due to the chase and escape force the chasers tend to move towards targets and thus the system exhibits complex collective motion, i.e. collective motion of both the target and chasers is coupled. To quantize the observed collective motion we employ the order parameter, 


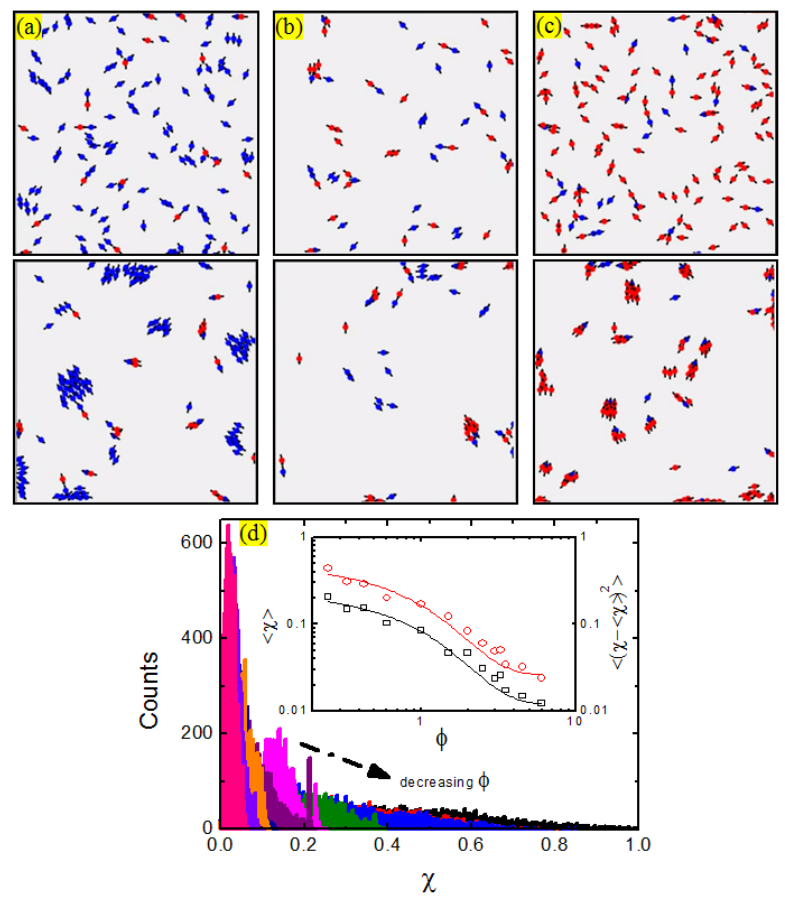

Fig. 1. (Color online) (a-c) Screenshots of the simulation for different concentration ratio $\phi$; (a) $\phi<1$, (b) $\phi=1$, and (c) $\phi>1$. The blue particles correspond to the targets while the red particles correspond to the chasers. The upper images correspond to the initial distribution of the particles while the lower images correspond to the steady state (after 1500 simulation runs). (d) Histogram of $\chi_{\text {chaser }}$ for various $\phi$. Inset. The mean and standard deviation of the $P\left(\chi_{\text {chaser }}\right)$ is plotted versus $\phi$. Solid line is an exponential decay fit.

$\chi$, given by, $\chi=\frac{1}{N v_{0}}\left|\sum_{j} \vec{v}_{j}\right|$, where $N$ is the total number of targets or chasers and $\vec{v}_{j}$ is the individual velocity of either a target or a chaser. Particles are all aligned when $\chi=1$ and there are no particle orientational correlation for $\chi=0$. In Figure 1.d we plot the distribution of $\chi_{\text {chaser }}$ for different $\phi$. From the distribution of $\chi_{\text {chaser }}$, statistical measures such as the mean order parameter, $\langle\chi\rangle$, and the standard deviation $\left\langle(\chi-\langle\chi\rangle)^{2}\right\rangle$ were extracted. Both the $\langle\chi\rangle$ and $\left\langle(\chi-\langle\chi\rangle)^{2}\right\rangle$ were found to be exponentially decaying function of $\phi$.

Our result may have some biological implications, for instance we observe that at higher concentration of targets, chasers chase mostly by themselves and not in herds as seen by the decrease of $\chi_{\text {chaser }}$. However for the case of lower concentration of targets, chasers chase mostly in groups. This behavior of food search is similar to that used by Dictyostelium at environments of low food concentration [7].

\section{Guided trapping of target SPPs}

The interaction between the SPP (target or chaser) and the V-shaped trap is such that only the SPP velocity component parallel to the trap contributes. Figure 2.a-g 

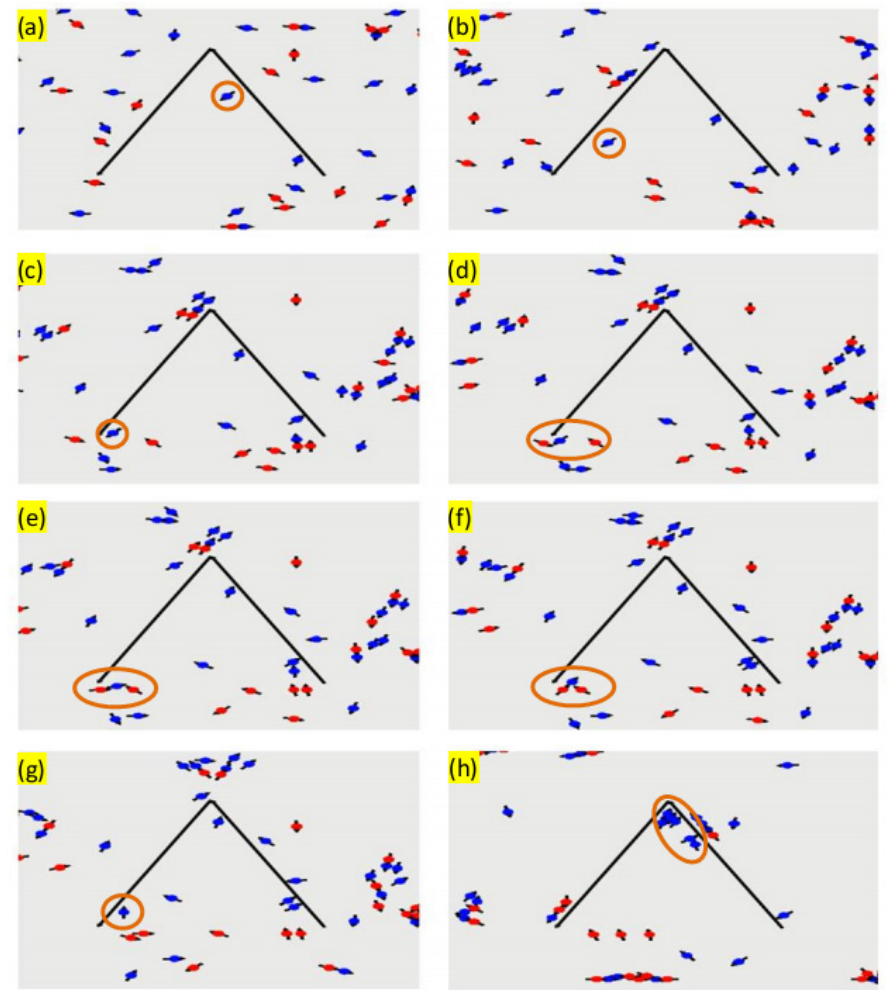

Fig. 2. (Color online) (a-g) Sequential screenshot images of the guided trapping of a target to a V-shaped trap for $\phi=1$. (h) Trapping at long time.

are simulation screenshots showing one mechanism of a guided trapping of a target SPP to a V-shaped trap for $\phi=1$. In Figure 2.a-c, the target which initially is located inside the trap has its direction of motion going outside the trap. We note that since there is no other target SPP's near it (no Vicsek interaction) and since no chaser is allowed inside the trap, our target of interest moves in a straight line until it encounters a chaser that causes it to move in the opposite direction. Finally in Figure 2.e-f we see the joint effect of two chasers that cause the target to return inside the trap. We observed that at long time, more target SPP's are trapped inside the trap although their trapping mechanism could be different from the one detailed above. Factors affecting the efficiency of the trapping mechanism such as noise, apex angle of the trap as well as the concentration ratio will be discussed in detail in a separate article [8].

\section{Conclusion}

The guided trapping of target SPP to a V-shaped trap is possible as verified via Brownian dynamic simulation. This trapping mechanism is robust as it can work 
on target SPPs of varying shapes and propulsion mechanism since these parameters were not specified in the simulation.

\section{References}

1. S. J. Ebbens and J. R. Howse, In pursuit of propulsion at the nanoscale, Soft Matter 6, 726 (2010).

2. P. Galajda, J. Keymer, P. Chaikin, and R. Austin, A wall of funnels concentrates swimming bacteria, J. Bacteriol. 189, 8704 (2007).

3. A. Kaiser, H. Wensik, and H. Löwen, How to Capture Active Particles, Phys. Rev. Lett. 108, 268307 (2012).

4. C. Reichhardt and C. J. Oslon Reichhardt, Active Matter Ratchets with an External Drift, arXiv:1307.0755v1

5. L. Angelani, Collective Predation and Escape Strategies, Phys. Rev. Lett. 109, 118104 (2012).

6. T. Vicsek, A. Czirok, E. Ben-Jacob, I. Cohen, and C. Shochet, Novel Type of Phase Transition in a System of Self-Driven Particles, Phys. Rev. Lett. 75, 1226 (1995).

7. B. A. Lazazzera, Quorum sensing and starvation: signals for entry into stationary phase, Current Opinion in Microbiology 3, 177 (2000).

8. R. Lacubtan and M. N. Confesor, in preparation. 